\title{
Ideal sinks are not always ideal: radiation damage accumulation in nanocomposites
}

\author{
Blas Pedro Uberuaga*, Samrat Choudhury, Alfredo Caro \\ Materials Science and Technology Division, Los Alamos National Laboratory, Los Alamos, NM, USA 87545
}

\begin{abstract}
Designing radiation tolerant materials is one of the primary challenges associated with advanced nuclear energy systems. One attractive route that has received much attention world-wide is to introduce a high density of sinks, often in the form of interfaces or secondary phases. Here, we develop a simple model of such nanocomposites and examine the ramifications of various factors on the overall radiation stability of the material. In particular, we determine how the distribution of secondary phases, the relative sink strength of those phases, and the irradiation temperature influence the radiation tolerance of the matrix. We find that the best scenario is one in which the sinks have intermediate strength, transiently trapping defects before releasing them back into the matrix. Neither perfect sinks nor the complete absence of sinks perform as well. This provides new insight into the optimal properties of nanocomposites for radiation damage environments.
\end{abstract}

Keywords: radiation damage, nanocomposite, computer modeling

\section{Introduction}

One of the significant technological challenges limiting the further adoption of nuclear energy production, be it fission or fusion, is the durability of structural materials

5 such as the cladding exposed to harsh irradiation environ- ${ }_{35}$ ments. Significantly more energy could be extracted from the fuel in a fission reactor if the cladding could sustain greater amounts of damage. Currently, the fuel is removed after only $4-6 \%$ of the uranium atoms in the $\mathrm{UO}_{2}$ fuel 10 are "burned", leaving the great majority of the fuel as waste [1]. One of the reasons for this is that the cladding material surrounding the fuel degrades with time, in great part due to the radiation damage accumulation experienced by the material. Thus, there is great impetous to develop materials with enhanced radiation tolerance.

A number of approaches have been proposed and pursued to develop radiation-resistant materials [2]. One approach is to use materials that are inherently radiation tolerant. For example, bcc metals tend to exhibit superior tolerance against radiation damage as compared to fcc metals [2]. Another approach is to provide a high density of sinks for radiation-induced defects. Sinks can take various forms, including pre-existing dislocation structure [3], grain boundaries and interfaces [4-10], and distributed nanoparticles such as those present in oxide dispersion strengthened steels [11]. This last approach is particularly intriguing as it can in principle combine both high ${ }^{55}$ sink density with radiation tolerant materials, as the origin of the sinks can be particles of highly resistant phases

*Corresponding author: Phone: 505-667-9105

Email address: blas@lanl.gov (Blas Pedro Uberuaga) the presence of dispersed nanoparticles can indeed increase the overall radiation tolerance of a composite [11], the role that the properties of the second phase have in dictating the enhanced radiation tolerance is still unclear. In particular, how do the properties of defects within the second phase, relative to the primary matrix phase, determine the overall stability of the composite is still an open question.

In a recent paper [12], we examined how the defect content near a heterophase interface depends on the relative thermodynamic and kinetic properties of defects within the two phases. A one-dimensional model that qualitatively reproduced the observations from a series of experiments [7-10] showed that significant changes in the defect accumulation near the interface could occur even though the interface itself did not trap defects. That is, the interface simply acted as a transition from one material to the next and changes in defect accumulation were driven by changes in defect migration rates as they crossed the interface. In that paper, we suggested that secondary phases in nanocomposites could be used as sinks to trap defects and improve the overall radiation tolerance of the matrix. In this paper, we extend that model to two dimensions and explore the impact that secondary phases have on radiation damage in nanocomposites.

\section{Methodology}

The methodology for this study is essentially the same as presented in Ref. [12], simply extended to two dimensions. We are solving the reaction-diffusion system defined 
by the following two equations:

$$
\begin{aligned}
c_{I}^{j}(t)= & c_{I}^{j}(t-\Delta t)+S_{I}-K_{V I} c_{I}^{j}(t-\Delta t) c_{V}^{j}(t-\Delta t) \\
& +\sum_{m} c_{I}^{m}(t-\Delta t) k_{I}^{m \rightarrow j} \\
& -\sum_{m} c_{I}^{j}(t-\Delta t) k_{I}^{j \rightarrow m} \\
c_{V}^{j}(t)= & c_{V}^{j}(t-\Delta t)+S_{V}-K_{V I} c_{I}^{j}(t-\Delta t) c_{V}^{j}(t-\Delta t) \\
& +\sum_{m} c_{V}^{m}(t-\Delta t) k_{V}^{m \rightarrow j} \\
& -\sum_{m} c_{V}^{j}(t-\Delta t) k_{V}^{j \rightarrow m}
\end{aligned}
$$

where the equations are discretized on a grid with cells (or grid points) of size $\Delta x=\Delta y$. Here, $c_{X}^{j}$, with $X=I$ or $V$, is the concentration of interstitials and vacancies, respectively, in cell $j . m$ are the neighboring cells - in the case of two dimensions, $m=4 . S_{X}$ and $K_{V I}$ are the scaled defect production rate and the scaled rate $\mathrm{co}^{-}{ }_{100}$ efficient for interstitial-vacancy recombination. They are related to base rates via $S_{X}=S_{X}^{0} \Delta t$ and $K_{V I}=K_{V I}^{0} \Delta t$. $k_{X}$ are diffusion rates for for interstitials and vacancies and are related to base diffusion rates via $k_{X}=\frac{\Delta t}{\Delta x^{2}} k_{X}^{0}$, where $k_{X}^{0}=k_{X 0} \exp \left(-E^{m} / k_{B} T\right)$. Here, $k_{B}$ is the Boltz- ${ }_{105}$ mann constant, $T$ is the temperature, and $k_{X 0}$ is a diffusion prefactor. $E^{m}$ is the migration barrier for the defect, modified by $\mu$ when the neighboring cell is of a different 70 phase; see Fig. 1 for details.

In this work, for all simulations presented, $k_{X 0}=4.2 \times_{110}$ $10^{-2} \mathrm{~cm}^{2} / \mathrm{s}$. To facilitate comparisons between simulations, all simulations were performed with $\Delta x=1 \mathrm{~cm}$. The time step $\Delta t$ was chosen to ensure stability in the 75 equations, and thus had to satisfy the condition that it was smaller than $1 / 4$ of the fastest rate in the model. For ${ }_{115}$ safety, we chose it to be 0.22 times the inverse of the fastest rate for most cases, though in a few simulations even a more conservative time step was chosen.

The choice of $k_{X 0}$ in principle defines the length and time scales of the model. However, given the qualitative ${ }_{120}$ nature of this study and the fact that the actual time and length scales of the simulation are coupled via the factor $\Delta t / \Delta x^{2}$, we will not focus on the particular time 85 and length scales of the simulations. In addition, the interstitial-vacancy recombination rate in this model is in- ${ }_{125}$ dependent of temperature; in reality, it is a function of the mobility of the defects. The fact that in this model the recombination rate is independent of the temperature 90 means that changing the temperature is akin to changing the length scales of the model. We will thus view tempera- ${ }_{130}$ ture and length scale interchangeably. Of course, a length scale of $1 \mathrm{~cm}$ is not nanoscale by any measure. However, as mentioned below, as a consequence of the fact that $K_{I V}$ 95 is a constant independent of length scale and temperature (because it is independent of $k_{X}$ ), the simulations become ${ }_{135}$ increasingly difficult to converge as $\Delta x$ is reduced. Thus, here, we focus on (unrealistically) high temperatures as an

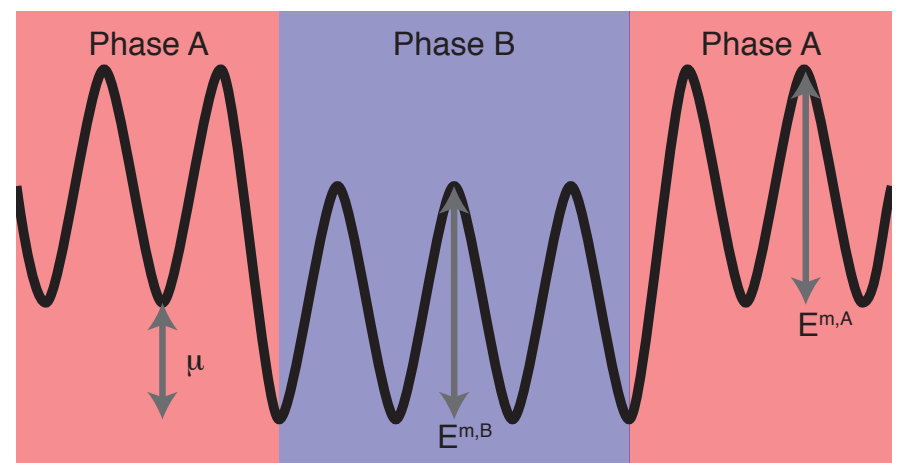

Figure 1: Schematic of the landscape for defects in the model used in this work. Defect energetics are defined by the migration energy $E^{m}$ in each of the two phases, A and B, as well as the difference in formation energy or chemical potential for the defect between phases $\mathrm{A}$ and $\mathrm{B}, \mu$. Similar landscapes are defined for interstitials and vacancies.

analog for nanoscale lengthscales. In the end, whether the model behaves as a nanocomposite depends on the mobility of vacancies over the length scale of the problem; hence, raising the temperature has the qualitative effect of simulating a nanoscale regime. This could be remedied by making $K_{I V}$ dependent on $k_{X}$, but that would also eliminate the ability to have a common reference result for all of the simulations.

The system of equations described above were evolved until steady state was reached. Steady state was defined by the criterion that no concentration in any cell changed by more than $10^{-6 \%}$ between time steps. Tests indicate that further convergence (to $10^{-7 \%}$ ) did not significantly change the concentrations.

The equations were solved for two-dimensional morphologies that consisted of two phases. Any cell could be one of two phases: matrix A or secondary phase B. Thus, the migration energies of interstitials and vacancies in $\mathrm{A}$ versus $B$ and the relative chemical potentials of interstitials and vacancies in A versus B define the model, as discussed in Ref. [12]. These are represented by the variables $E_{X}^{m}$ and $\mu_{X}$, where $\mu_{X}$ is the difference in chemical potential of defect $X$ in phase B as compared to phase A (a positive value represents a preference for phase $\mathrm{B}$ ). The landscape for this model is schematically shown in Fig. 1.

Unless otherwise stated, $S_{X}^{0}=10^{-6} \mathrm{~cm}^{-2} \mathrm{~s}^{-1}$ and $K_{I V}^{0}=$ $10^{-7} \mathrm{~cm}^{2} \mathrm{~s}^{-1}$ for all simulations. The system of equations described above can be solved analytically for the steady state concentration for a case in which there is no phase $\mathrm{B}$ [3]. In that case, the steady-state concentrations for both interstitials and vacancies are equal to $\sqrt{S_{X} / K_{I V}}$ which, for the values used here, is $\sqrt{10}=3.16$ defects/unit area. We note that there is an error in the values for these rates given in Ref. [12]. The values were given as $S=0.001$ $\mathrm{cm}^{-3} \mathrm{~s}^{-1}$ and $K_{I V}=0.001 \mathrm{~cm}^{3} \mathrm{~s}^{-1}$ for the majority of simulations when in reality they were $S=10^{-7} \mathrm{~cm}^{-3} \mathrm{~s}^{-1}$ and $K_{I V}=10^{-7} \mathrm{~cm}^{3} \mathrm{~s}^{-1}$.

Further, most of the simulations were performed for 
the following conditions: $T=1000 \mathrm{~K}, E_{I}^{m}=0.5 \mathrm{eV}$ in both phases, $E_{V}^{m}=1.0 \mathrm{eV}$ in both phases, $\mu_{V}=2.0 \mathrm{eV}$, and $\mu_{I}=2.0 \mathrm{eV}$. In some of the cases, some of these variables were explicitly modified to understand the impact of that variable on the defect accumulation in the ${ }^{195}$ system, as described below. These choices for the migration energies of vacancies and interstitials are arbitrary, but are representative of real materials. Typically, interstitial migration is much faster than vacancy migration, as represented by these values. Further, in fcc and bcc met-200 als, vacancy migration barriers are on the order of $0.5-1.0$ $\mathrm{eV}$ while interstitial migration energies are roughly $0.01-$ 0.3 [13]. Interestingly, in urania, the primary fuel form in most fission reactors, the values are swapped, and oxygen vacancies migrate with an energy of roughly $0.5 \mathrm{eV}_{205}$ while oxygen interstitials migrate with an energy of about $1.0 \mathrm{eV}$; the cation values are much higher [14, 15]. Thus, while these values do not represent any particular material, they are representative of the values for migration energies typical of many materials.

We use vacancy accumulation in phase $\mathrm{A}$ as a measure of the radiation tolerance of the model composite. As interstitials diffuse faster, they are more likely to leave the are interested in how adding a secondary phase to a ma-215 trix can be used to modify the radiation tolerance of the matrix, so we are not concerned with the defect evolution in phase B at this time, for simplicity. In addition, there is an extremely large phase space of variables to examine and we admit we have only studied a small slice through that 220 phase space. The average vacancy concentration in phase A, then, is taken as our metric of radiation tolerance and is defined as the concentration of vacancies in each cell of phase A, averaged over all cells of phase A. Phase B is not considered in this averaging.

Finally, for the majority of results presented, we consider a random distribution of phase $\mathrm{B}$ within phase $\mathrm{A}$. The simulations were performed on a $20 \times 20$ discretized unless otherwise noted. To ensure that the results presented were not sensitive to the particular random dis-230 tribution considered, we performed multiple simulations in which a new random distribution was generated for each point, typically for a total of 5 simulations for each case 180 considered. In a small number of cases, particularly those reported in Fig. 3, we performed 50 simulations to ensure 235 that there were no statistical outliers in the system. We have performed tests on a $100 \times 100$ sample to further ensure our results are not artifacts of the simulation size.

\section{Results}

Solving the system of equations described above, we examine how various properties of the nanocomposite, including the phase fraction and distribution of phase $\mathrm{B}$ within phase $\mathrm{A}$ and the values of $E_{X}^{m}$ and $\mu_{X}$, impact ${ }_{245}$ is that the radiation tolerance of phase A can be modified by creating a composite with phase B. A schematic of the effective landscape for defects is provided in Fig. 1.

\subsection{Composite Morphology}

We begin by examining how the distribution of phase $B$ within phase A influences the defect accumulation in phase A. Figure 2 illustrates the types of morphologies we have considered, which represent distributed particles and layered (line) morphologies. In the case of the distributed particle morphology, we have examined two distributions, an ordered array of points and a random distribution. In the case of the point geometry in Fig. 2a, the separation between different cells of phase B is maximized. For the line geometry in Fig. 2b, phase B is distributed in lines or layers, mimicking the effect of a multilayer geometry. Finally, Fig. 2c shows a random distribution of phase B within A, meant to represent an arbitrary composite structure.

The average steady state concentrations of vacancies in phase $\mathrm{A}$ as a function of both the morphology of the model composite and the fraction of phase $\mathrm{B}$ within the composite is shown in Fig. 3. Overall, the steady state concentrations as a function of the fraction of phase B follow the same trend regardless of the detailed arrangement of phase B. At a fraction of phase B $\chi=0$, the model reproduces the analytical result (as shown in the inset). Once a small amount of $\mathrm{B}$ is added to the composite, the vacancy concentrations become much larger, over an order of magnitude larger, than the pure phase A case $(\chi=0)$. It is only when $\chi 0.25-0.5$, depending on the composite morphology, does the vacancy concentration in the matrix A approach the $\chi=0$ value.

There is an initial spike in the accumulation of vacancies which occurs for low phase fractions of $\mathrm{B}$, with a peak at about $\chi=0.0002$ for the current parameters, as highlighted in the inset in Fig. 3. The initial spike in concentration in vacancies as the fraction of phase B is increased, followed by the subsequent reduction, is a consequence of the difference in sink behavior of phase B for interstitials and vacancies. Recall that the migration energy of interstitials in this model is $0.5 \mathrm{eV}$ while that for vacancies is 1.0 $\mathrm{eV}$. Thus, interstitials diffuse much more quickly and are able to find the phase B sinks much more efficiently. The vacancies do not have high enough diffusivities to reach the phase B sinks if those sinks are of low density. Thus, for small $\chi$, interstitials are removed from the matrix by the sinks but vacancies are unable to reach those sinks and defect recombination in the matrix is reduced, leading to higher concentrations of vacancies. In contrast, as $\chi$ increases, the ability of vacancies to find sinks increases and the vacancy accumulation in the matrix is reduced. Of course, the magnitude of this bias will depend on the relative diffusion distance of vacancies versus the spacing between phase B sinks, which depends both on temperature and the length scale of the microstructure, as discussed below. 


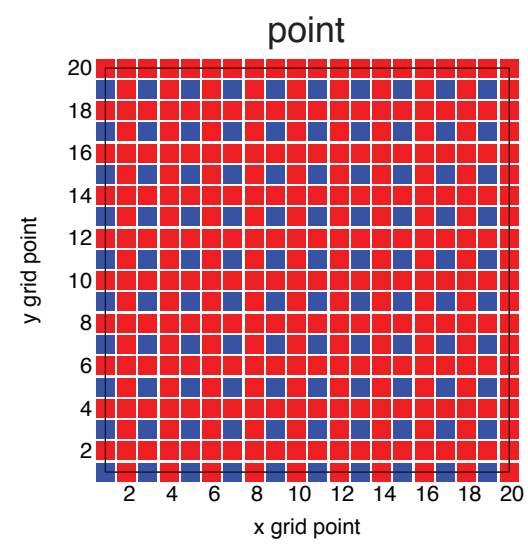

(a)

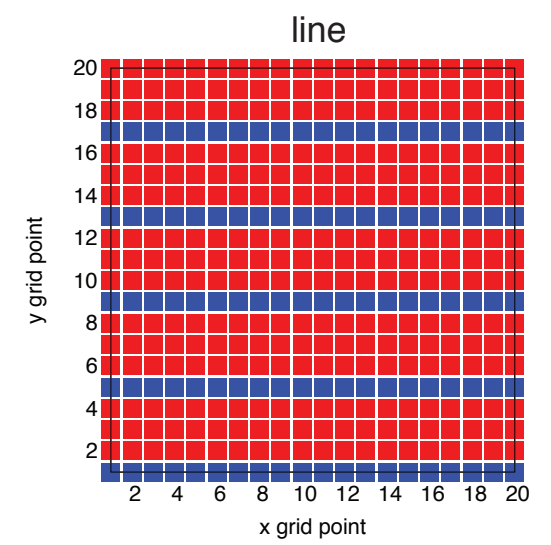

(b)

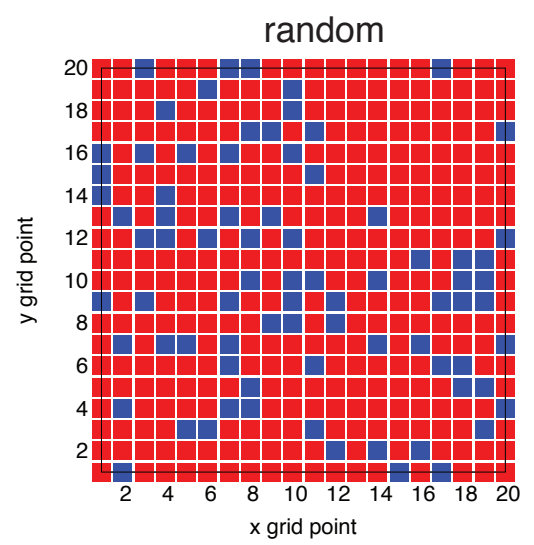

(c)

Figure 2: Representative illustrations of the different types of morphologies considered in this study, which represent different distributions of phase B within phase A: (a) isolated points, (b) lines, and (c) random. The fraction of phase B in these examples is $0.25,0.25$, and 0.2 , respectively. The color scheme is red for phase A and blue for phase B.

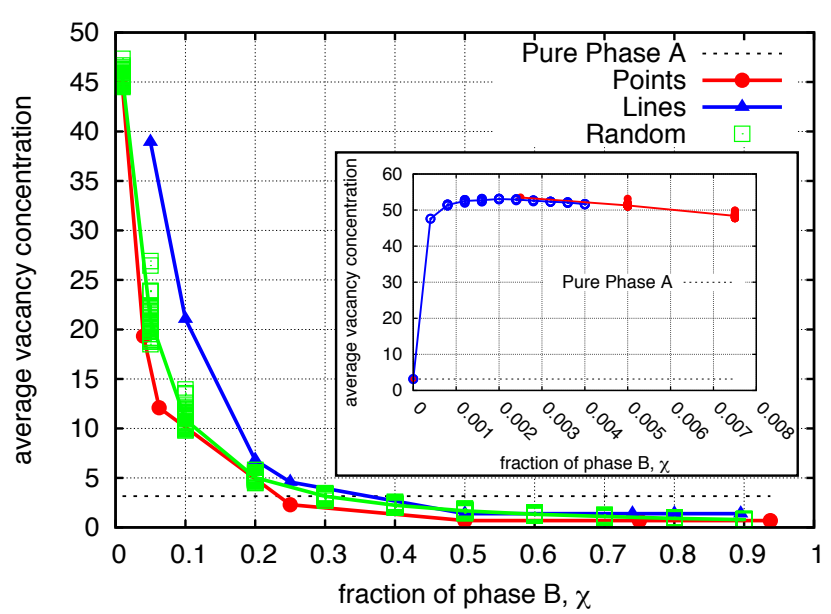

Figure 3: Average concentration of vacancies in phase $\mathrm{A}$ as a function of the fraction of phase $\mathrm{B}$ at $T=1000 \mathrm{~K}$ for three different types of distribution of phase $\mathrm{B}$ in $\mathrm{A}$. In this and subsequent figures, the points represent results from individual simulations while the lines go through the average of those points. In the case of the random distribution, 50 independent simulations were done for each value of the fraction of phase B. For reference, the concentration for a system in which no phase B is present, a case for which there is an analytical solution, is provided as a reference in this and subsequent figures. The inset magnifies the behavior for fractions of phase $B$ near 0 , where there is a peak in the vacancy accumulation. Open ${ }^{280}$ symbols are for simulations on a $50 \times 50$ grid, closed symbols are for simulations on a $20 \times 20$ grid.

While the manner in which phase B is distributed in phase A does not change the overall trends, it does influ- ${ }^{285}$ ence the response of the system quantitatively. Typically, the point geometry results in lower vacancy accumulation than the random geometry, which is lower than the line geometry. This is a consequence of the average distance between a cell containing phase $\mathrm{A}$ to a cell containing phase $\mathrm{e}^{290}$ the peometry, this distance is minimized as no $\mathrm{B}$ cells are neighbors and all B cells are surrounded by A cells. As we move to a random and then line morphology, this becomes less and less true; more B cells neighbor other $\mathrm{B}$ cells. Thus, the ability of vacancies to diffuse from phase ${ }_{260}$ A to phase $\mathrm{B}$ depends on the manner in which phase $\mathrm{B}$ is distributed. The ability of the secondary phase to increase the radiation tolerance of the composite increases as phase $\mathrm{B}$ is more dispersed. Essentially, this is a surface/volume effect and, as might be expected, the best case scenario is when the surface area is maximized.

\subsection{Temperature Effects}

As mentioned earlier, because of how we are treating interstitial-vacancy recombination in this model (it is independent of the defect mobilities and thus temperature), 270 changing the temperature of the simulation has a similar effect as changing the length scale of the model. That is, both increasing the temperture or reducing the length scale increase the effective diffusion length of vacancies relative to the length scale of the microstructure and increase the ability of vacancies to find phase $\mathrm{B}$ sinks. The one major difference is that, with a change in temperature, the effective strength of the phase B sinks is reduced, while that strength would remain constant if the length scale were simply changed. That said, we focus on temperature effects here because, as discussed below, length scale effects are harder to examine.

Figure 4 illustrates this effect for cases in which the distribution of phase $\mathrm{B}$ within phase $\mathrm{A}$ is randomly determined. As the temperature is reduced from 1000 to 900 $\mathrm{K}$, the vacancy accumulation increases and the amount of phase B needed to recover the tolerance of the pure A system is also increased. However, as the temperature is increased, two notable changes arise. First, temperatures greater than $1500 \mathrm{~K}$, all composites, regardless of the fraction of phase B, exhibit lower levels of vacancy accumulation than the pure phase A case. However, composites with larger amounts of phase B perform worst at the highest temperature than they do at intermediate temperature 


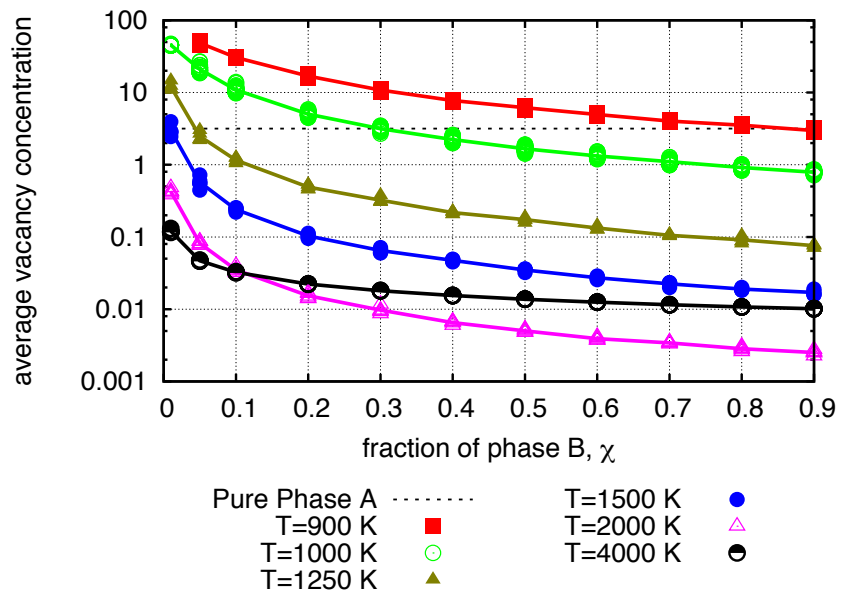

Figure 4: Average concentration of vacancies in phase $\mathrm{A}$ as a function of the fraction of phase $\mathrm{B}$ and temperature.

(compare $T=4000 \mathrm{~K}$ with $T=2000 \mathrm{~K}$ in Fig. 4). At the

highest temperature, the sinks are not as strong, relatively, and there is a competition between diffusion and reemission from the sinks, as discussed in more detail below.

We have stated that, in this model, raising the temperature is roughly analogous to refining the microstructure/reducing the length scale of the simulation. We have not been able to extensively test assertion this as changing the length scale via $\Delta x$ dramatically increases the time needed to converge the simulations and, in particular, simulations truly representing a nanoscale length scale require immense computer time. This is likely a consequence of the fact that in this model $K_{I V}$ is a constant regardless of length scale or temperature. However, we have tested the role of microstructural length scale on the response of the composite under irradiation and the results are provided in Fig. 5. This figure does demonstrate that by reducing the length scale associated with the composite, the radiation tolerance, measured as the average vacancy concentration in phase $\mathrm{A}$, is increased, similarly as raising the temperature. In fact, comparing with Fig. 4, reducing the length scale by a factor of 10 is roughly equivalent to increasing the temperature by about $500-1000 \mathrm{~K}$. Thus, as one would expect, the performance is very sensitive to length scale. ${ }_{335}$

\subsection{Trapping Effects}

In this model, the ability of cells of phase B to trap defects is a function of the chemical potential difference for defects in phase $\mathrm{A}$ relative to phase $\mathrm{B}: \mu_{X}=\mu_{X}^{A}-\mu_{X}^{B}{ }^{\cdot 340}$ If $\mu_{X}>0$, defect $X$ is attracted to phase B. Conversely, it is repelled by phase B. Figure 6 shows the consequence of changing the relative strength of phase B sinks for interstitials. For strong sink strength, or large $\mu_{I}$, the phase $\mathrm{B}$ sinks are detrimental, at least for small $\chi$, for damage ${ }_{345}$ accumulation as discussed above. They trap interstitials, but by doing so prevent recombination with sluggish vacancies. However, as the sink strength is reduced, or as $\mu_{I}$

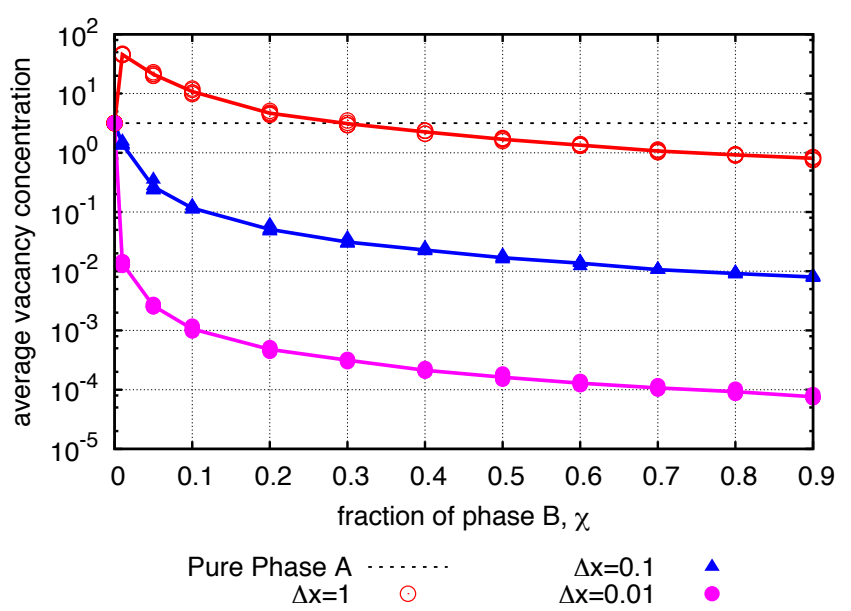

Figure 5: Average concentration of vacancies in phase A as a function of the fraction of phase $\mathrm{B}$ and $\Delta x$.

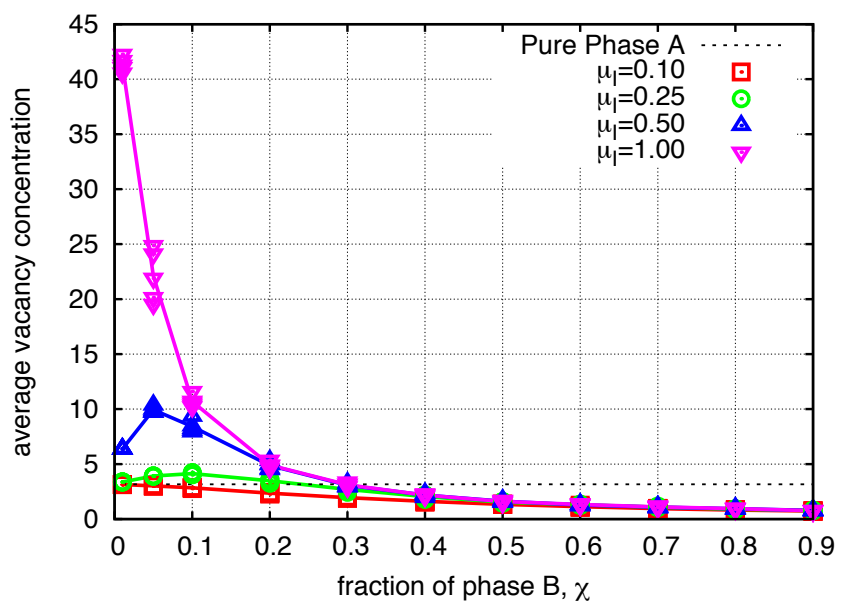

Figure 6: Average concentration of vacancies in phase A as a function of the fraction of phase $\mathrm{B}$ and the chemical potential difference for interstitials $\mu_{I}$ between phase $\mathrm{B}$ and phase $\mathrm{A}$.

decreases. When the attraction of interstitials to phase B is particularly weak $\left(\mu_{I}=0.10\right)$, the introduction of phase $\mathrm{B}$ into the composite always leads to a decrease in vacancy accumulation, relative to the pure phase A reference.

Figure 7 shows the interstitial and vacancy concentrations in each cell of the simulation for two different chemical potentials for interstitials $\left(\mu_{I}=1.0\right.$ and 0.1$)$ for a case in which the fraction of phase $\mathrm{B} \chi=0.1$. Figures $7 \mathrm{a}-\mathrm{b}$ show the concentration maps for $\mu_{I}=1.0$. In this case, because the phase B traps are strong and the interstitials are fast, the interstitials quickly get absorbed by phase B sites and the overall interstitial content is low. As a consequence, the vacancy concentrations are relatively high as there are simply no interstitials to annihilate the vacancies. However, when the chemical potential is decreased to $\mu_{I}=0.1$, as shown in Figs. 7c-d, the interstitial content is higher and the vacancy content is lower. Neither are as high, on average, as for the case when no phase B is 

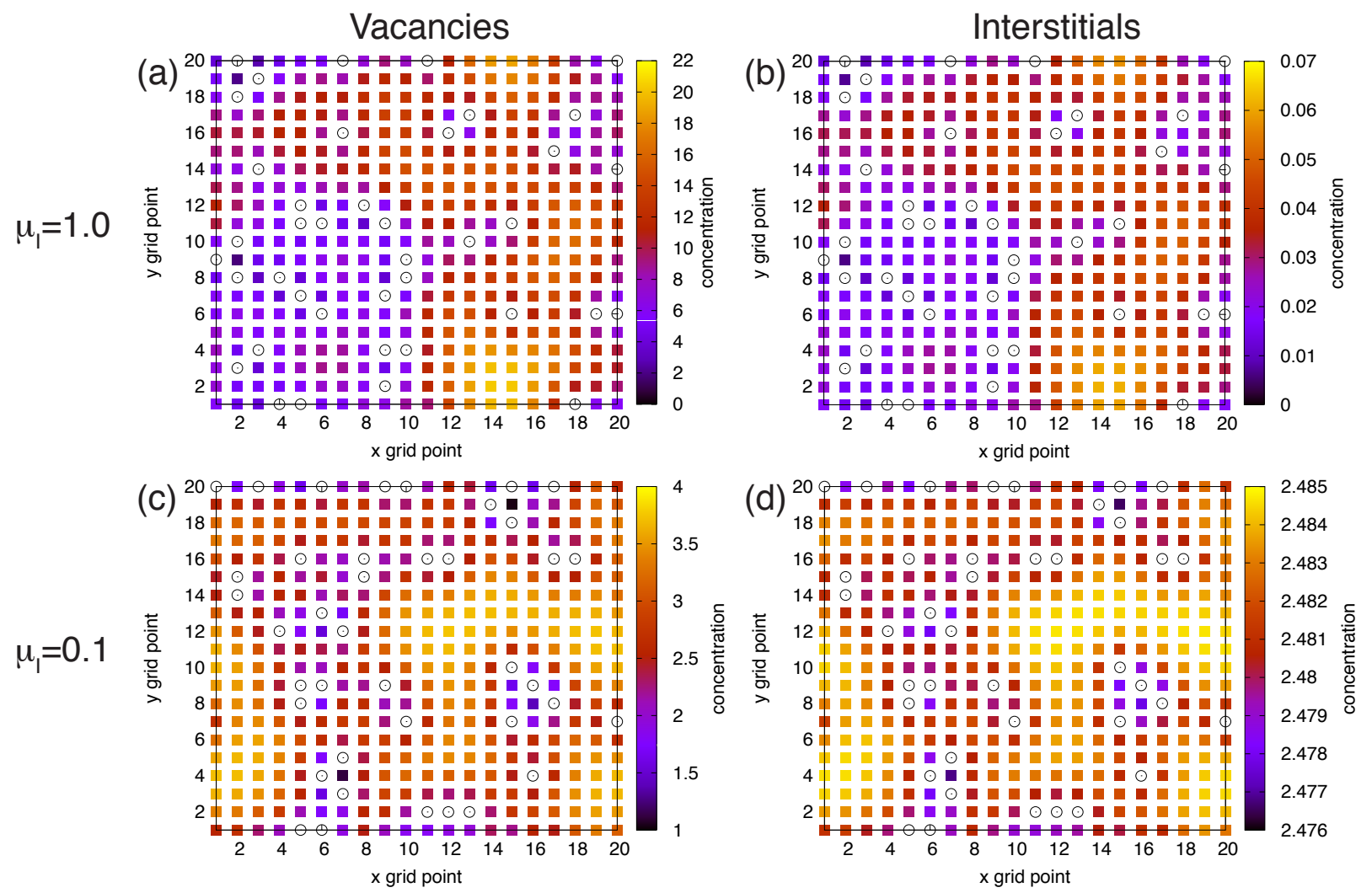

Figure 7: Concentration maps for both interstitials and vacancies as a function of position and the chemical potential of interstitials. In these figures, cells containing phase B are represented by open circles.

present.

One could imagine two explanations for this result. First, it must be recalled that defect production is occurring in both phases simultaneously and at the same rate in this model. It is possible that phase $B$ acts as a source of interstitials, via the radiation-induced generation 380 interstitials in phase B, that then escape and annihilate vacancies in phase $\mathrm{A}$. This possibility can be eliminated, however, by performing simulations (not shown) in which the defect production rate in phase $\mathrm{B}$ is set to zero. The defect content in phase $\mathrm{A}$ is unchanged in this scenario. The $\mathrm{T}_{385}$ A migrate to phase B and are momentarily trapped, but later reemitted back into the matrix A. At steady state, this means that, because the sink is weak, there is a back flux of interstitials into the matrix, which leads to greater 390 rates of interstitial/vacancy annihilation there.

Why, however, would this lead to a net reduction in defect content as compared to the pure phase A simulations, in which the interstitials are never trapped in the first place? The answer is revealed in Fig. 8 in which the $e_{39}$ 30 average concentration of vacancies in phase $\mathrm{A}$ is plotted as a function of the chemical potential of vacancies $\mu_{V}$ for a case where the chemical potential of interstitials $\mu_{I}=0.1$. The original case shown in Fig. 6 corresponds to the curve in which $\mu_{V}=2.0$. Here, we see a net reduction in the va-400
375 cancy concentration as compared to the case of pure phase A (labeled "Pure Phase A"). When $\mu_{V}=0$, the overall vacancy concentration increases, exceeding the pure phase A result. If $\mu_{V}$ is reduced even further to -2.0 , the vacancy content rises dramatically with an increase in the content of phase $\mathrm{B}$, which occurs because vacancies generated in $\mathrm{B}$ are expelled into $\mathrm{A}$. These results indicate that the overall reduction in vacancy concentration seen for the case of $\mu_{V}=2.0$ and $\mu_{I}=0.1$ is a consequence of two factors. First, interstitials are trapped by and then reemitted from sites containing phase B. At the same time, vacancies are slowly migrating to regions of phase $\mathrm{B}$, though much more sluggishly than interstitials. Thus, phase B both traps vacancies and acts a source for interstitials, which increase the overall efficiency of annihilation in phase A. If vacancies are not attracted to the traps, vacancies accumulate in phase A faster. Similarly, if interstitials are not reemitted (per Fig. 6), annihilation in phase A is less efficient. Annihilation is maximized when interstitials are reemitted from and vacancies are attracted to traps.

Figure 7 also reveals denuded zones, or defect depletion zones, near regions containing phase $\mathrm{B}$. This is particularly pronounced for in Figs. 7a-c. In regions of the matrix A that are farthest from any cell containing B, the defect concentrations reach a maximum. This is consistent with many results that show, for example, void denuded zones 


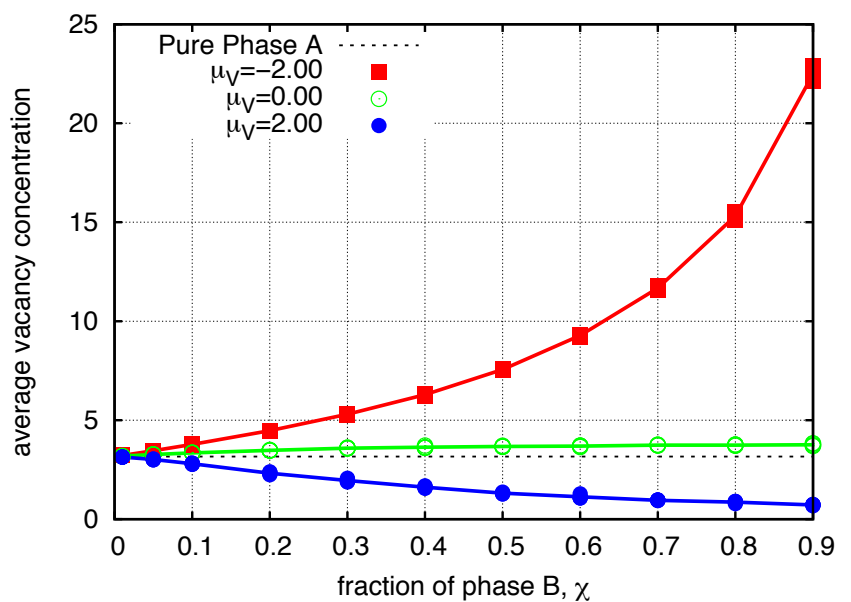

Figure 8: Average concentration of vacancies in phase A as a function ${ }^{450}$ of the fraction of phase $B$ and the chemical potential difference for vacancies $\mu_{V}$ between phase $\mathrm{B}$ and phase $\mathrm{A}$, for a case where $\mu_{I}=$ 0.1 .

near sinks $[4,5]$.

\section{Discussion}

The results presented above offer several intriguing insights into the behavior of nanocomposites under irradiation. First, the inclusion of small amounts of sinks, represented here by the concentration or fraction of phase B, does not always increase the tolerance of the composite. In many cases, it will degrade radiation tolerance. This depends on the relative diffusion distance of vacancies. If vacancies can easily reach the sinks, the sinks will lead to enhanced radiation tolerance; otherwise, they will worsen it. This is not a surprise. As discussed in Ref. [2], if there is a significant bias in the sink strength for interstitials ${ }_{470}$ versus vacancies, vacancy supersaturations can occur. In the model presented here, such a bias occurs of the difference in mobility between vacancies and interstitials is high, a difference that is reduced with increasing temperature. This result emphasizes the fact that increased sink density is not always beneficial. Particularly at lower temperatures, increased sink density can be detrimental [16].

The second important insight is that ideal sinks may be worse for increasing the overall radiation tolerance of a composite than weak strengths. That is, rather than having perfect or no sinks, it may be optimal to have weak trix. Our results indicate that the greatest reduction in defect accumulation occurs for just such a scenario. In the model presented here, interstitial reemission is independent of vacancy concentrations, but we have seen in istic simulations that such reactions can be further facilitated by the nearby presence of vacancies [17, 18], enhancing even more the annihilation near the sink.

Interestingly, the details of the distribution of phase $B$ within phase $\mathrm{A}$ do not make a qualitative difference in the
435 behavior of the composite, though the levels of defect accumulation do differ when the morphology is changed from a point-like distributed geometry to a layered geometry. A layered geometry offers less overall benefit as compared to a point geometry, since the average distance between 40 defects and the phase B sinks is larger in this case. This suggests that (a) maximal benefit is obtained by dispersing the secondary phase as finely as possible within the matrix and (b) layered geometries, used as models in many studies [7-10, 19-21], provide qualitatively the same behavior as three dimensional nanocomposites and are thus suitable replacements for such geometries.

Of course, most structural materials in nuclear applications operate at temperatures at which vacancies are highly mobile and thus increasing sink density will increase radiation tolerance and it would be preferable to have perfect sinks to capture all defects. This is true of $\mathrm{Fe}$ and $\mathrm{Ni}$ based alloys, for example (see Fig. 9 in Ref. [2]). However, for some materials, such as the oxides (e.g. $\mathrm{UO}_{2}$ ) that make up the fuel itself in which the disparity between vacancy and interstitial mobilities might even be reversed, or in laboratory settings in which the temperatures may be significantly lower, the optimum microstructure might be very different, and the optimum types of sinks might also be very different. In particular, weaker sinks may afford maximum enhancement of radiation tolerance.

It should be noted that this model, while offering qualitative insight into the behavior of composites under irradiation, certainly has several limitations. First, interstitials and vacancies within the two phases are indistinguishable, beyond the different migration energies and chemical potentials. While this might be an appropriate model for a material in which the two phases have the same chemistry (such as the fcc and bcc phases of Fe in a duplex steel), in reality most composities will contain phases with different chemistries. Thus, migration of interstitials and vacancies across the interface will necessarily lead to intermixing, which is neglected here. Further, no special properties of the interfaces themselves are accounted for. Most interfaces will themselves serve as traps for defects [20, 22-24], complicating the defect evolution near the interface. In addition, under most realistic radiation conditions, defect clusters will be formed (either directly in-cascade or via thermal aggregation) which would lead to a new suite of defects to interact with the sinks. Finally, as the defect content evolves, if it reaches large enough concentrations, new phenomena such as amorphization [25-29] or void nucleation $[4,6]$ will occur. All of these effects are neglected in this model. However, we expect that the general insights provided by this work are general and apply not only to nanocomposites, but to any material containing sinks.

It should be noted that a number of factors determine the ultimate response of the composite to radiation damage accumulation. Not one or a small set of parameters determine that response. Rather, all of the factors influence the radiation tolerance of the material, including the mobility of each type of defect, the relative strength of the 


\section{natorial explosion in terms of correlating behavior with ${ }^{565}$ parameters.}

Finally, while this model provides intriguing new insight into the behavior of nanocomposites under irradiation, it requires experimental validation. To the best studies of the behavior of nanocomposites under irradiation as a function of the phase composition. In previous work, we examined the effect of radiation damage on the morphological evolution at interfaces in bi-layers [7-

$\left.\begin{array}{ll}525 & 10\end{array}\right]$ and this model qualitatively reproduced the physical trends observed in those experiments [12]. However, ex-575 actly how the nature of secondary phases would influence radiation damage evolution in the matrix of a nanocomposite is still an open question that requires further ex-

${ }_{530}$ perimental work. In particular, experiments in which the ${ }^{580}$ matrix phase is constant over a series of composite structures in which the secondary phase is changed, and, for example, the amorphization susceptibility of the matrix is monitored as a function of the secondary phases would be $e^{555}$ ideal. The Mg-Ti-O system may be suitable for this purpose, as there are a number of phases in this system. Such a study would validate the basic physical trends identified in this manuscript.

\section{Conclusions}

Using a two-dimensional reaction-diffusion model, we ${ }^{595}$ examine various aspects of a nanocomposite under irradiation. The nanocomposite is modeled as a mixture of two phases in which the properties of defects (migration ${ }_{600}$ energies and chemical potentials) differ. The simulation results lead to the following two conclusions:
- Adding sinks to a material does not necessarily increase radiation tolerance. If the sinks do not interact with both interstitials and vacancies, sinks can degrade radiation tolerance. So-called dislocation bias is one such scenario [30].

- More interestingly, in some conditions, ideal sinks may be worse than no sinks at all. However, weak sinks, because of the reemission of defects, can lead to an overall increase in radiation tolerance compared to either of the other cases.

These results provide new insight into the behavior of nanocomposites under irradiation as well as new avenues for designing radiation tolerant materials.

\section{Acknowledgments}

The authors gratefully acknowledge support by the Center for Materials at Irradiation and Mechanical Extremes, an Energy Frontier Research Center funded by the U.S. Department of Energy, Office of Science, Office of Basic Energy Sciences under Award Number 2008LANL1026. Los Alamos National Laboratory, an affirmative action equal opportunity employer, is operated by Los Alamos National Security, LLC, for the National Nuclear Security Administration of the U.S. DOE under contract DE-AC5206NA25396. We acknowledge helpful discussions with E. Martínez.

\section{References}

[1] S. J. Zinkle, G. S. Was, Materials challenges in nuclear energy, Acta Materialia 61 (2013) 735-758. doi:10.1016/j.actamat.2012.11.004.

[2] S. J. Zinkle, L. L. Snead, Designing radiation resistance in materials for fusion energy, Annual Review of Materials Research 44 (2014) 241-267. doi:10.1146/annurev-matsci-070813-113627.

[3] G. S. Was, Fundamentals of Radiation Materials Science, Springer, Berlin, 2007.

[4] R. W. Siegel, S. M. Chang, R. W. Balluffi, Vacancy loss at grain-boundaries in quenched polycrystalline gold, Acta Metall. 28 (1980) 249-257.

[5] R. Yamada, S. J. Zinkle, G. P. Pells, Defect formation in ionirradiated al2o3 and mgal2o4 - effects of grain-boundaries and fusion transmutation products, J. Nucl. Mater. 191 (1992) 640644.

[6] W. Z. Han, M. J. Demkowicz, E. G. Fu, Y. Q. Wang, A. Misra, Acta Materialia 60 (2012) 6341.

[7] M. Zhuo, E. Fu, L. Yan, Y. Wang, Y. Zhang, R. Dickerson, B. Uberuaga, A. Misra, M. Nastasi, Q. Jia, Interface-enhanced defect absorption between epitaxial anatase tio2 film and single crystal srtio3, Scripta Materialia 65 (9) (2011) 807 - 810.

[8] M. Zhuo, B. P. Uberuaga, L. Yan, E. G. Fu, R. M. Dickerson, Y. Q. Wang, A. Misra, M. Nastasi, Q. Jia, Radiation damage at the coherent anatase $\mathrm{TiO} 2 / \mathrm{SrTiO} 3$ interface under $\mathrm{Ne}$ ion irradiation, J. Nucl. Mater. 429 (2012) 177-184.

[9] Z. Bi, B. P. Uberuaga, L. J. Vernon, E. Fu, Y. Wang, N. Li, H. Wang, A. Misra, Q. X. Jia, Radiation damage in heteroepitaxial BaTiO3 thin films on $\mathrm{SrTiO} 3$ under Ne ion irradiation, J. Appl. Phys. 113 (2013) 023513.

[10] Z. Bi, B. P. Uberuaga, L. J. Vernon, J. A. Aguiar, E. Fu, S. Zheng, S. Zhang, Y. Wang, A. Misra, Q. X. Jia, J. Appl. Phys. 115 (2014) 124315. 
[11] G. R. Odette, M. J. Alinger, B. D. Wirth, Recent developments in irradiation-resistant steels, Annu. Rev. Mater. Res. 38 471503.

[12] B. P. Uberuaga, E. Martinez, Z. Bi, M. Zhuo, Q. X. Jia, M. Nastasi, A. Misra, A. Caro, Materials Research Letters 1 (2013) 193-199.

[13] C.-C. Fu, J. D. Torre, F. Willaime, J.-L. Bocquet, A. Barbu, Nature Materials 4 (2005) 68.

[14] B. Dorado, J. Durinck, P. Garcia, M. Freyss, M. Bertolus, J. Nucl. Mater. 400 (2010) 103.

[15] D. A. Andersson, P. Garcia, X.-Y. Liu, G. Pastore, M. Tonks, P. Millett, B. Dorado, D. R. Gaston, D. Andrs, R. L. Williamson, R. C. Martineau, B. P. Uberuaga, C. R. Stanek, J. Nucl. Mater. 451 (2014) 225.

[16] Y. Chimi, A. Iwase, N. Ishikawa, M. Kobiyama, T. Inami, S. Okuda, Accumulation and recovery of defects in ionirradiated nanocrystalline gold, J. Nucl. Mater. 297 (2001) 355357.

[17] X.-M. Bai, A. F. Voter, R. G. Hoagland, M. Nastasi, B. P. Uberuaga, Science 327 (5973) (2010) 1631-1634.

[18] I. J. Beyerlein, A. Caro, M. J. Demkowicz, N. A. Mara, A. Misra, B. P. Uberuaga, Materials Today 16 (2014) 443

[19] A. Misra, M. J. Demkowicz, X. Zhang, R. G. Hoagland, JOM 59 (2007) 62.

[20] M. J. Demkowicz, R. G. Hoagland, J. P. Hirth, Interface structure and radiation damage resistance in cu-nb multilayer nanocomposites, Phys. Rev. Lett. 100 (2008) 136102.

[21] W. Han, M. J. Demkowicz, N. A. Mara, E. Fu, S. Sinha, A. D. Rollett, Y. Q. Wang, J. S. Carpenter, I. J. Beyerlein, A. Misra, Advanced Materials 25 (2013) 6975-6979.

[22] X.-Y. Liu, B. P. Uberuaga, M. J. Demkowicz, T. C. Germann, A. Misra, M. Nastasi, Phys. Rev. B 85 (2012) 012103.

[23] M. A. Tschopp, K. N. Solanki, F. Gao, X. Sun, M. A. Khaleel, M. F. Horstemeyer, Physical Review B 85 (6) (2012) 064108.

[24] P. P. Dholabhai, J. A. Aguiar, A. Misra, B. P. Uberuaga, J. Chem. Phys. 140 (2014) 194701.

640 [25] M. Lang, J. Lian, J. Zhang, F. Zhang, W. J. Weber, C. Trautmann, R. C. Ewing, Phys. Rev. B 79 (2009) 224105.

[26] K. E. Sickafus, L. Minervini, R. W. Grimes, J. A. Valdez, M. Ishimaru, F. Li, K. J. McClellan, T. Hartmann, Radiation tolerance of complex oxides, Science 289 (2000) 748-751.

27] K. E. Sickafus, R. W. Grimes, J. A. Valdez, A. Cleave, M. Tang, M. Ishimaru, S. M. Corish, C. R. Stanek, B. P. Uberuaga, Radiation-induced amorphization resistance and radiation tolerance in structurally related oxides, Nat. Mater. 6 (2007) 217223.

[28] Y. H. Li, B. P. Uberuaga, C. Jiang, S. Choudhury, J. A. Valdez, M. K. Patel, J. Won, Y. Q. Wang, M. Tang, D. J. Safarik, D. D. Byler, K. J. McClellan, I. O. Usov, T. Hartmann, G. Baldinozzi, K. E. Sickafus, Phys. Rev. Lett. 108 (2012) 195504.

[29] J. A. Aguiar, P. P. Dholabhai, Z. Bi, Q. X. Jia, E. Fu, Y. Q. Wang, T. Aoki, J. Zhu, A. Misra, B. P. Uberuaga, Adv. Mater. Interfaces 1 (2014) 1300142.

[30] G. W. Greenwood, A. J. E. Foreman, D. E. Rimmer, J. Nucl. Mater. 4 (1959) 305. 